\title{
Thyroid and iodine nutritional status: a UK perspective
}

\author{
Author: Mark Vanderpump ${ }^{A}$
}

Iodine is an essential component of the thyroid hormones, which play a crucial role in brain and neurological development. At least one-third of the world's population is estimated to be iodine deficient predominantly in developing countries. Recently concern had also been expressed about the iodine status in industrialised countries such as the UK. A recent survey of the UK iodine status found that that more than two-thirds of schoolgirls aged 14-15 years were iodine deficient due to the reduced milk intake. Maternal iodine deficiency in pregnancy is correlated with cognitive outcomes such as intelligence quotient and reading ability in offspring. No randomised trial data exist for iodine supplementation in mild-moderate iodine-deficient pregnant women. It is possible to combine population interventions to reduce population salt intake with salt iodisation programmes in order to maintain adequate levels of iodine nutrition.

KEYWORDS: Iodine, thyroid, iodine deficiency, urinary iodine, cognition, salt iodisation

\section{The importance of iodine}

Iodine is an essential component of the thyroid hormones thyroxine (T4) and triiodothyronine (T3) produced by the thyroid gland, which play a crucial role in brain and neurological development. The ideal dietary allowance of iodine recommended by the World Health Organization (WHO) is $150 \mu \mathrm{g}$ iodine per day, which increases to $200-250 \mu \mathrm{g}$ per day in pregnancy. ${ }^{1}$ Iodine deficiency is recognised as a global problem with large populations at risk who are living in an environment where the soil has been deprived of iodine. This arises from past glaciation, compounded by the leaching effects of snow, water and heavy rainfall that remove iodine from the soil. The mountainous regions of Europe, the Northern Indian Subcontinent, the extensive mountain ranges of China, the Andean region in South America and the lesser ranges of Africa are all iodine deficient. ${ }^{2}$ In 1980 the WHO estimated that at least one-third of the world's population was iodine deficient, predominantly those living in developing countries. ${ }^{3}$

Endemic delayed motor and mental development has long been recognised to be associated with iodine deficiency.

Author: Aconsultant physician, Department of Endocrinology, Royal Free London NHS Foundation Trust, London, UK
Severe iodine deficiency may be associated with impairment in the psycho-neurological outcome in the progeny because both mother and offspring are exposed to iodine deficiency during gestation (and the postnatal period). Controlled studies performed in iodine-deficient regions have confirmed that iodine supplementation eliminated new cases of delayed motor and mental development reduced infant mortality and improved cognitive function in the general population. ${ }^{4}$ Even mild iodine deficiency is thought to lead to reductions of 10-15 in intelligence quotient (IQ) points.

Iodine deficiency remains a huge problem and many people are still deficient despite major national and international efforts to increase iodine intake, primarily through the voluntary or mandatory iodisation of salt. The WHO estimates that two billion people, including 285 million school-age children, still have iodine deficiency, defined as a urinary iodine (UI) excretion of less than $100 \mu \mathrm{g} / 1 .{ }^{5,6}$ Recent epidemiological data suggest that iodine deficiency may also now be an emerging issue in industrialised countries such as the UK, previously thought of as iodine-sufficient. ${ }^{7}$ International efforts to control iodine deficiency are slowing and reaching the onethird of the worldwide population that remains deficient poses major challenges.

\section{Epidemiological criteria for assessing iodine nutrition}

The size of the thyroid gland changes inversely in response to alterations in iodine intake, with a lag interval that varies from a few months to several years. Goitre prevalence is an index of the degree of longstanding iodine deficiency and is less sensitive than UI in the evaluation of a recent change in the status of iodine nutrition. However, the evaluation of goitre prevalence based on palpation has been questioned because the reproducibility of assessment by palpation is low, especially with the size estimation of smaller glands, particularly in children. Therefore, the method of choice is now ultrasonography, which is reproducible with a maximum deviation of $10 \%$. The prevalence of goitre in iodine-replete populations is below $5 \% .^{1,8}$

UI excretion is a good marker of the recent dietary intake of iodine over days and is therefore the index of choice for evaluating the degree of iodine deficiency. Levels have been expressed per gram of creatinine excretion but more recent studies have indicated that the creatinine level is variable depending on the general nutritional status of the population and that this contributes an independent source of variation that invalidates the ratio. In addition, urinary creatinine also 


\section{Table 1. The spectrum of iodine deficiency disorders.}

$\begin{array}{ll}\text { Fetus } & \text { Abortions } \\ & \text { Stillbirths } \\ & \text { Congenital anomalies } \\ & \text { Increased perinatal mortality } \\ & \text { Endemic delayed motor and mental development } \\ \text { Neonate } & \text { Neonatal goitre } \\ & \text { Neonatal hypothyroidism } \\ & \text { Endemic mental retardation } \\ & \text { Increased susceptibility of the thyroid gland to } \\ & \text { nuclear radiation } \\ \text { Child and } & \text { Goitre } \\ \text { adolescent } & \text { (Subclinical) hypothyroidism } \\ & \text { Impaired mental function } \\ & \text { Retarded physical development } \\ & \text { Increased susceptibility of the thyroid gland to } \\ \text { nuclear radiation } & \text { Goitre with its complications } \\ \text { Hypothyroidism } & \text { Impaired mental function } \\ \text { Spontaneous hyperthyroidism in the elderly } & \text { Iodine-induced hyperthyroidism } \\ \text { nuclear radiation }\end{array}$

decomposes after three days without refrigeration, whereas UI remains stable for months. The WHO/International Council for the Control of the Iodine Deficiency Disorders (ICCIDD)/ United Nations Children's Fund (UNICEF) recommend that for national, school-based surveys of iodine nutrition, the median UI from a representative sample of spot urine collections from 1,200 children (30 sampling clusters of 40 children per cluster) can be used to classify a population's iodine status. ${ }^{9}$ For epidemiological studies, a population distribution of UI is required rather than individual levels. Because the frequency distribution is usually skewed towards elevated values, the median is considered instead of the mean as indicating the status of iodine nutrition. Table 1 shows the epidemiological criteria presently recommended for assessing iodine nutrition based on median UI concentrations in school-age children. UI values can be most conveniently expressed as a range with a median or by the proportions at a series of cut off points, $<20 \mu \mathrm{g} / \mathrm{l},<50 \mu \mathrm{g} / \mathrm{l}$ and $<100 \mu \mathrm{g} / \mathrm{l}$.

Elevated serum thyrotrophin (TSH) is the most sensitive indicator of the potential risk of iodine deficiency on brain development but is only elevated in at least moderate iodine deficiency. Serum T4 and T3 are even less specific indicators of iodine deficiency because they are modified usually only in conditions of severe iodine deficiency and are not recommended in routine assessment and monitoring. ${ }^{8}$ Serum thyroglobulin represents a sensitive index of a state of thyroid hyperstimulation and whole blood from finger pricks spotted on filter paper cards can determine serum TSH and serum thyroglobulin. However, due to technical and financial limitations these are only rarely used.

\section{Iodine deficiency disorders}

Epidemiological studies have demonstrated that reduced iodine intake during pregnancy leads to goitrogenesis, lower free T4 concentrations and increased serum TSH in pregnant women. ${ }^{1}$ The main change in thyroid function associated with the pregnant state is the requirement for an increased production of thyroid hormone that depends directly upon the adequate availability of dietary iodine and the underlying integrity of the thyroid. Physiological adaptation can take place when the iodine intake is adequate. When iodine intake is deficient, pregnancy can reveal an underlying iodine restriction.

The term iodine deficiency disorders (IDD) refers to all the ill-effects of iodine deficiency in a population that can be prevented by ensuring that the population has an adequate intake of iodine (Table 2). ${ }^{1}$ Severe iodine deficiency results in severe learning disabilities, deafness and impaired motor development. The effects of mild-to-moderate iodine deficiency on cognition are less well known than those of moderate-tosevere deficiency but it is assumed that there is a continuum of

Table 2. Epidemiological criteria presently recommended for assessing iodine nutrition based on median UI concentrations in school-age children. ${ }^{9}$

\begin{tabular}{|c|c|c|}
\hline Median UI $\mu \mathrm{g} / \mathrm{I}$ & Iodine intake & Iodine nutrition \\
\hline$<20$ & Insufficient & Severe iodine deficiency \\
\hline $2-49$ & Insufficient & Severe iodine deficiency \\
\hline 50-99 & Insufficient & Severe iodine deficiency \\
\hline 100-199 & Adequate & Optimal \\
\hline 200-299 & More than adequate & $\begin{array}{l}\text { Risk of iodine-induced hyperthyroidism within } 5-10 \text { years following introduction of } \\
\text { iodised salt in susceptible }\end{array}$ \\
\hline$\geq 300$ & Excessive & $\begin{array}{l}\text { Risk of adverse health consequences (iodine-induced hyperthyroidism, autoimmune } \\
\text { thyroid diseases) }\end{array}$ \\
\hline
\end{tabular}

UI = urinary iodine 
disability with more subtle impairments of IQ and motor ability associated with less severe deficiency.

In countries which have previously been considered iodine sufficient, such as the US, iodised salt is used in about $70 \%$ of households. However, recent data have shown that the median UI excretion in adults declined from $320 \mu \mathrm{g} / \mathrm{l}$ in 1971-74 to $145 \mu \mathrm{g} / \mathrm{l}$ in 1988-94, and was more recently measured at $168 \mu \mathrm{g} / \mathrm{l}$ in $2001-2002 .{ }^{10} \mathrm{Up}$ to $15 \%$ of women of childbearing age, and almost $7 \%$ of them during a pregnancy, had iodine excretion levels in the range of moderate iodine deficiency, namely below $50 \mu \mathrm{g} / \mathrm{l}$. The most recent US survey data from 2009-2010 demonstrated that the US population remains iodine-sufficient, with a median UI concentration of $144 \mu \mathrm{g} / \mathrm{l}$ but that the median UI concentration among pregnant women had dropped to less than $150 \mu \mathrm{g} / \mathrm{l}$, indicating mild iodine deficiency. Particular attention is therefore required to ensure that pregnant women receive an adequate iodine supply by administering multivitamin tablets containing iodine supplements, in order to achieve the WHO recommended dietary allowance of 200-250 $\mu \mathrm{g}$ iodine/day.

Mild to moderate iodine deficiency occurs in areas that are not immediately recognised as iodine deficient. Although the introduction of iodised salt has considerably improved the situation globally in the developing world, iodine deficiency remains an issue in continental Europe where it is estimated that up to $50 \%$ of children live in iodine deficient communities. ${ }^{11}$ The iodine intake may vary markedly within a country because of significant variations in the natural iodine content of food and water. This was demonstrated in Denmark where pregnant women without iodine supplements had a median iodine excretion level of $66 \mu \mathrm{g} / \mathrm{g}$ creatinine in Copenhagen and $33 \mu \mathrm{g} / \mathrm{g}$ creatinine in East Jutland. ${ }^{12}$

As maternal T4 is crucial to fetal nervous system maturation, even modest states of iodine deficiency could be deleterious. Data from the US, Netherlands and Tasmania suggest that the children of women with hypothyroxinaemia may have psycho-neurological deficits and delayed mental and motor function when compared with controls. ${ }^{13-15}$ This correlates with the studies in classic areas of iodine deficiency where a range of psychological and neurological deficits in children have been described during the past century, ${ }^{4}$ but it is maternal hypothyroxinaemia rather than high serum TSH that is the clear abnormality. Thus areas previously thought to be iodine sufficient, such as Australia and New Zealand, now have evidence of substantial gestational iodine deficiency, which may lead to low maternal circulating T4 concentrations. ${ }^{16}$

There were few data of the consequences of mild to moderate iodine deficiency in older children. A recent randomised, placebocontrolled, double-blind trial in 184 children aged $10-13$ years in New Zealand (median UI $63 \mu \mathrm{g} / \mathrm{l}$ ) had demonstrated that iodine supplementation ( $150 \mu \mathrm{g} / \mathrm{l}$ daily) for 28 weeks improved perceptual reasoning, suggesting that mild iodine deficiency may prevent children attaining their full intellectual potential. ${ }^{17}$ Similar positive findings have been observed in European children who were moderately iodine deficient. ${ }^{18}$

\section{The re-emergence of iodine deficiency in the UK}

Iodine deficiency has historically been considered an issue for developing countries rather than industrialised countries such as the UK. Endemic goitre associated with iodine deficiency was at

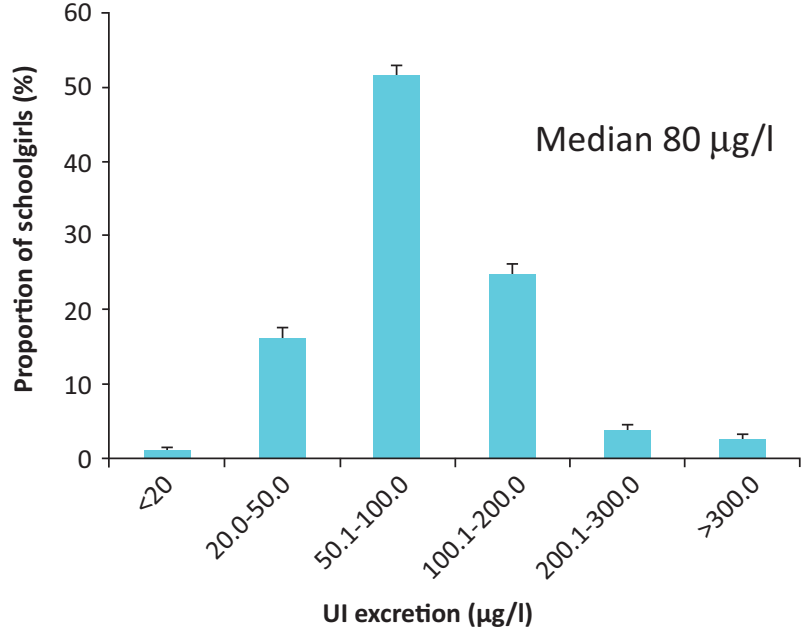

Fig 1. UI concentrations in UK schoolgirls $(n=737)$ showing the proportions of participants with mild (50-99 $\mu \mathrm{g} / \mathrm{l})$, moderate $(20-49 \mu \mathrm{g} / \mathrm{l})$, and severe $(<20 \mu \mathrm{g} / \mathrm{l})$ iodine deficiency. ${ }^{21} \mathrm{UI}=$ urinary iodine.

one time widespread in the UK and Medical Research Council surveys in 1924 and 1944 reported visible goitre in up to $50 \%$ of adult women and schoolgirls. ${ }^{19}$ No salt iodisation programme was adopted in the UK, unlike in other European countries. Since the 1940s, significant changes in farming practice in the UK were associated with a rise in the iodine content of milk, particularly during winter months when cattle are dependent on iodine-rich artificial feed. In addition, successive UK governments from the 1940s encouraged increased milk consumption in schoolchildren. By the 1980s this resulted in the iodine content of milk alone being almost sufficient to meet the recommended daily requirement of $150 \mu \mathrm{g}$ per day and has been described as an 'accidental public health triumph'. ${ }^{19}$ Although national monitoring of milk iodine content continued, the major concern was to avoid the harmful effects of iodine toxicity rather than deficiency.

Recently, concern has also been expressed concerning the finding that up to $50 \%$ of UK women of child-bearing age screened in smaller studies at single centres were iodinedeficient. ${ }^{20}$ A study of the iodine content of 36 different salt preparations from nine major national supermarkets in Cardiff identified iodine concentrations which varied from undetectable in 32 samples to trace quantities in two. Only two samples contained meaningful concentrations of iodine $(20 \mathrm{mg} / \mathrm{kg})$ related to the prevention of iodine deficiency. A similar pattern of iodine deficiency has been seen in Ireland, with iodine intake being particularly low in the summer months. ${ }^{20}$

A systematic survey of the UK iodine status in 2009 found that the median UI excretion in a sample of 737 schoolgirls was $80 \mu \mathrm{g} / \mathrm{l}$ and that more than two-thirds had a UI of less than $100 \mu \mathrm{g} / \mathrm{l}$ (Fig 1). ${ }^{21}$ The study focused on young female subjects aged 14-15 years who are pre-pregnancy, as in the short to medium term it is their children that are most susceptible to the adverse effects of iodine deficiency. Milk intake was positively associated with UI (Fig 2) and as milk iodine concentrations were unchanged it was likely to be the reduced amount of milk now drunk by the population responsible for the decline in iodine status. 


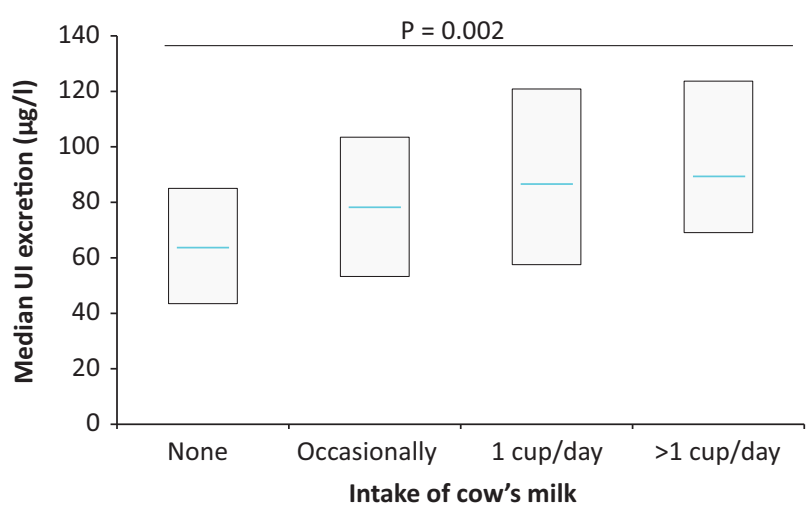

Fig 2. Increasing intake of milk is independently associated with higher median UI in survey of UK iodine status in schoolgirls $(p=0.002) .{ }^{21} \mathrm{UI}=$ urinary iodine.

The Avon Longitudinal Study of Parents and Children (ALSPAC) study has related maternal iodine deficiency in pregnancy from stored urine iodine samples to cognitive outcomes, such as IQ and reading ability in offspring up to the age of nine years. ${ }^{22}$ This study recruited pregnant women in the early 1990s from the south-west UK, collected data during pregnancy and followed the health and development of their children. The women were classified as being iodine deficient by WHO criteria (median UI concentration $92 \mu \mathrm{g} / \mathrm{g}$ creatinine). Children born to mothers broadly classified as iodine deficient $(<150 \mu \mathrm{g} / \mathrm{g}$ creatinine) based on WHO criteria were more likely to have scores in the lowest quartile for total IQ and reading accuracy even after adjustment for 21 potential confounders, including maternal education, age and breastfeeding.

\section{Correction of iodine deficiency}

The findings of the UK survey have major public health importance as the UK is now number eight on the list of the top 10 iodine-deficient countries (based on national median UI less than $100 \mu \mathrm{g} / \mathrm{l}$ in children), with the greatest numbers of schoolage children with insufficient iodine intake in $2011 .{ }^{23}$ Iodine deficiency in an industrialised country presents a significant challenge, as recently addressed in Australia. ${ }^{24}$ Despite the clear benefits in correcting iodine deficiency, a fear of iodineinduced thyroid dysfunction has at times delayed or limited the implementation of iodine supplementation in regions with iodine deficiency. In adults, mild iodine deficiency is associated with a decreased risk of overt and subclinical hypothyroidism, as well as autoimmune thyroiditis and an increased risk of non-toxic nodular goitre. ${ }^{1}$ A sudden increase in iodine supply to those in an iodine-deficient region may enhance thyroid autoimmunity through both a cellular and humoral immune response and may result in hypothyroidism in those with damaged thyroid glands, and hyperthyroidism in those with an underlying multinodular goitre or Graves' disease, although it is unlikely to do so if the deficiency is not severe and if the increase is relatively small.

Large epidemiological studies from Denmark have now carefully documented the pattern of thyroid disease after introduction of iodised salt in a mildly deficient population. ${ }^{25} \mathrm{An}$ initial voluntary iodine fortification programme proved ineffective so a mandatory iodine fortification programme of bread salt and household salt was introduced in 2000, aiming to increase the iodine intake in adults by an average of $50 \mu \mathrm{g}$ per day. This resulted in an increase in the population median UI concentrations from $53-68 \mu / 1$ in the original cohort to $93-108 \mu \mathrm{g} / \mathrm{l}$ when the cohort was reassessed five years later. Positive benefits both in terms of goitre prevalence and hyperthyroidism have been observed with small increases of both thyroid peroxidase antibodies and thyroglobulin antibody titres in all age groups, but most pronounced in young women. Other smaller cohorts studied suggest that this enhanced thyroid autoimmunity might be reversible and reduce over time. ${ }^{26}$ It was too early in the natural history of autoimmune thyroiditis to detect an impact in terms of clinically relevant hypothyroidism, but at five years a slightly higher serum TSH was seen in those subjects with positive anti-thyroid antibodies. ${ }^{25}$

\section{Conclusions}

In developed countries such as the UK there is a strong public health objective to lower salt intake to reduce the risk of hypertension. ${ }^{10}$ It is estimated that only $10 \%$ of UK salt intake is added to food at the table, so dialogue with the food and salt industries to establish the feasibility of adding iodised salt to processed food is essential. While accepting the available evidence-base for salt iodisation to correct iodine deficiency, ${ }^{7}$ a recent UK government scoping paper from the Standing Advisory Commission on Nutrition (SACN), which advises the chief medical officer, has not recommended iodine supplementation in pregnancy and even still questions the methodology of the iodine status assessment in populations used by the WHO.

For the government to consider iodine prophylaxis to offset the adverse effects of iodine deficiency, data are required to provide reassurance that at the population level the benefits far outweigh any disadvantages. Salt iodisation is safe, equitable, largely self-financing, and extremely cost effective in an industrialised country. ${ }^{7,10}$ The alternative strategy is daily oral potassium iodide supplements for the most susceptible groups, such as women pre-pregnancy. However, it is accepted in the UK that only $50 \%$ of pregnancies are planned and that iodine supplementation should ideally be at least three months prior to a pregnancy. ${ }^{27}$ No randomised trial data exist for iodine supplementation in mild-moderate iodine-deficient pregnant women. Recent epidemiological data may be the catalyst to stimulate interest and awareness of iodine deficiency and its correction as an issue of major public health importance in the UK. The ongoing monitoring of the population iodine status remains critically important, especially in those most at risk, such as young women. It is possible to combine population interventions to reduce population salt intake with salt iodisation programmes in order to maintain adequate levels of iodine nutrition.

\section{Conflict of interest}

Dr Vanderpump is the current UK representative of the International Council for the Control of the Iodine Deficiency Disorders and President of the British Thyroid Association.

\section{References}

1 Zimmermann MB. Iodine deficiency. Endocr Rev 2009;30:376-408.

2 Stanbury JB and Hetzel BS. Endemic goiter and cretinism: iodine nutrition in health and disease. New York: J Wiley, 1980. 
3 de Benoist B, McLean E, Andersson M et al. Iodine deficiency in 2007; global progress since 2003. Food Nutr Bull 2008;29:195-202.

4 Pharoah POD, Buttfield IH, Hetzel BS. Neurological damage to the fetus resulting from severe iodine deficiency during pregnancy. Lancet 1971;1:308-10.

5 WHO global database on iodine deficiency. Geneva: World Health Organization. Available online at www.who.int/vmnis/database/ iodine/iodine_data_status_summary/en/index.html [Accessed 24 October 2014].

6 Engle PL, Black MM, Behrman JR et al. Strategies to avoid the loss of developmental potential in more than 200 million children in the developing world. Lancet 2007;369:229-42.

7 Vanderpump MPJ. Iodine deficiency as a new challenge for industrialised countries: A UK perspective. Int J Epidemiol 2012;41:601-4.

8 WHO, UNICEF and ICCIDD. Assessment of the Iodine Deficiency Disorders and monitoring their elimination. 2nd ed. Geneva: WHO, 2001.

9 World Health Organization, United Nations Children's Fund, International Council for the Control of Iodine Deficiency Disorders. Assessment of iodine deficiency disorders and monitoring their elimination. 3rd ed. Geneva: WHO, 2007.

10 Pearce EN, Andersson M, Zimmerman MB. Global iodine nutrition: Where do we stand in 2013? Thyroid 2013;23:523-8.

11 Zimmerman MB, Andersson M. Prevalence of iodine deficiency in Europe in 2010. Ann Endocrinol (Paris) 2011;72:164-6.

12 Laurberg P, Pederson KM, Vestergaard H et al. High incidence of multinodular goitre in the elderly population in a low iodine intake area vs. high incidence of Graves' disease in the young in a high iodine area: comparative surveys of thyrotoxicosis epidemiology in East-Jutland Denmark and Iceland. J Int Med 1991;229:415-20.

13 Henrichs J, Bongers-Schokking JJ, Schenk JJ et al. Maternal thyroid function during early pregnancy and cognitive functioning in early childhood: the generation R study. J Clin Endocrinol Metab 2010;95:4227-34.

14 Pop VJ, Brouwers EP, Van Baar AL et al. Maternal hypothyroxinaemia during early pregnancy and subsequent child development: a 3-year follow-up study. Clin Endocrinol (Oxf) 2003;59:282-8.

15 Li M, Eastman CJ, Waite KV et al. Are Australian children iodine deficient? Results of the Australian National Iodine Nutrition Study. Med J Aust 2006;184:165-69.

16 Hynes KL, Otahal P, Hay I et al. Mild iodine deficiency during pregnancy is associated with reduced educational outcomes in the offspring: 9-year follow-up of the gestational iodine cohort. J Clin Endocrinol Metab 2013;98:1954-62.
17 Gordon RC, Rose MC, Skeaff SA et al. Iodine supplementation improves cognition in mildly iodine-deficient children. Am J Clin Nutr 2009;90:1264-71.

18 Zimmermann MB, Connolly K, Bozo M et al. Iodine supplementation improves cognition in iodine-deficient schoolchildren in Albania: a randomized, controlled, double-blind study. Am J Clin Nutr 2006;83:108-14.

19 Phillips DIW. Iodine, milk, and the elimination of endemic goiter in Britain: the story of an accidental public health triumph. J Epidemiol Community Health 1997;51:391-3.

20 Lazarus JH and Smyth PPA. Iodine deficiency in the UK and Ireland. Lancet 2008;372:888.

21 Vanderpump MP, Lazarus JH, Smyth PP et al. Iodine status of UK schoolgirls: a cross-sectional survey. Lancet 2011;377:2007-12.

22 Bath SC, Steer CD, Golding J et al. Effect of inadequate iodine status in UK pregnant women on cognitive outcomes in their children: results from the Avon Longitudinal Study of Parents and Children (ALSPAC) study. Lancet 2013;381:331-7.

23 Zimmerman M. Iodine deficiency uncovered in the UK. International Council for Control of Iodine Deficiency Disorders. IDD Newsletter Volume 39, No 3, August 2011. Available online at www.thyroid.org/ professionals/education/documents/IDD_NL_Aug11.pdf [24 October 2014].

24 Gallego G, Goodall S, Eastman CJ. Iodine deficiency in Australia: is iodine supplementation for pregnant and lactating women warranted? Med J Aust 2010;192:461-3.

25 Pedersen IB, Knudsen N, Carlé A et al. A cautious iodization program bringing iodine intake to a low recommended level is associated with an increase in the prevalence of thyroid autoantibodies in the population. Clin Endocrinol (Oxf) 2010;75:110-6.

26 Mazziotti G, Premawardhana LD, Parkes AB et al. Evolution of thyroid autoimmunity during iodine prophylaxis - the Sri Lankan experience. Eur J Endocrinol 2003;149:103-10.

27 Moleti M, Di Bella B, Giorgianni G et al. Maternal thyroid function in different conditions of iodine nutrition in pregnant women exposed to mild-moderate iodine deficiency: an observational study. Clin Endocrinol (Oxf) 2011;74:762-8.

Address for correspondence: Dr M Vanderpump,

Department of Endocrinology, Royal Free London NHS Foundation Trust, Pond Street, London, NW3 2QG, UK. Email: mark.vanderpump@nhs.net 